\title{
Elaboração de Suco Tropical de Umbu
}

\author{
Elaboration of Tropical Umbu Juice \\ Producción de Zumo de Umbu Tropical
}

Recebido: 17/02/2021 | Revisado: 23/02/2021 | Aceito: 19/04/2021 | Publicado: 02/05/2021

Aurianna Coelho Barros
ORCID: https://orcid.org/0000-0001-6747-9560
Instituto Federal de Educação, Ciência e Tecnologia do Sertão Pernambucano, Brasil
E-mail: aurianna1604@gmail.com
Helisvanhah Gretta Antunes Rodrigues Bispo
ORCID: https://orcid.org/0000-0003-0532-4285
E-mail: h.antunesbispo@gmail.com
Instituto Federal de Educação, Ciência e Tecnologia do Sertão Pernambucano, Brasil
Páulia Maria Cardoso Lima Reis
ORCID: https://orcid.org/0000-0003-1781-3924
Instituto Federal de Santa Catarina, Brasil
E-mail: pauliamaria.reis@gmail.com
Silvana Belém de Oliveira Vilar
ORCID: https://orcid.org/0000-0001-8141-3373
E-mail: silvana.belem@ifsertao-pe.edu.br
Instituto Federal de Educação, Ciência e Tecnologia do Sertão Pernambucano, Brasil
Nailton de Macedo Albuquerque Júnior
ORCID: https://orcid.org/0000-0001-6757-5114
Universidade Federal de Campina Grande, Brasil
E-mail: junior.nailton99@gmail.com
Iasmim Pereira Oliveira
ORCID: https://orcid.org/0000-0003-3971-3174
Instituto Federal de Educação, Ciência e Tecnologia do Sertão Pernambucano, Brasil
E-mail: mimuefs@gmail.com
Douglas Vinicius Pinheiro de Figueiredo
ORCID: https://orcid.org/0000-0002-1868-0927
Universidade Federal de Campina Grande, Brasil
E-mail: douglasdvpf@hotmail.com

\section{Resumo}

O Nordeste é uma região que se destaca como principal produtor de frutas no Brasil, com grande diversidade de frutas tropicais ainda pouco exploradas, como por exemplo o umbu que possui quantidades significativas de nutrientes, nesse contexto, o presente estudo teve como objetivo desenvolver um suco tropical de umbu da polpa congelada e do fruto in-natura. Todas as amostras foram submetidas as análises microbiológicas, por 90 dias, análises físico-químicas e a determinação de compostos bioativos, durante 120 dias de estocagem. Os resultados encontrados em ambos os sucos, apresentaram boas características microbiológicas, físico-químicas e nutritivas, no período de armazenamento estudado. Portanto, foram obtidos sucos de umbu com adequados parâmetros tecnológicos e alto potencial de valorização desta fruta regional, com características para atender a demanda crescente por alimentos nutritivos e com praticidade para o consumo.

Palavras-chave: Biodiversidade; Nutrientes; Compostos bioativos; Praticidade.

\begin{abstract}
The Northeast is a region that stands out as the main fruit producer in Brazil, with a great diversity of tropical fruits still little explored, such as umbu that has significant amounts of nutrients, in this context, this study aimed to develop a tropical juice of umbu from frozen pulp and fruit in-natura. All samples were submitted to microbiological analysis for 90 days, physical-chemical analysis and the determination of bioactive compounds during 120 days of storage. The results found in both juices, presented good microbiological, physicochemical and nutritional characteristics, during the studied storage period. Therefore, umbu juices were obtained with adequate technological parameters and high potential for valorization of this regional fruit, with characteristics to meet the growing demand for nutritious food and with practicality for consumption.
\end{abstract}

Keywords: Biodiversity; Nutrients; Bioactive compounds; Practicality.

\section{Resumen}

El Nordeste es una región que se destaca como principal productora de frutas en Brasil, con gran diversidad de frutas tropicales aún poco exploradas, como el umbu que posee importantes cantidades de nutrientes, en este contexto, este 
estudio tuvo como objetivo desarrollar un jugo tropical de umbu a partir de pulpa congelada y fruta in-natura. Todas las muestras se sometieron a análisis microbiológicos durante 90 días, a análisis físico-químicos y a la determinación de compuestos bioactivos durante 120 días de almacenamiento. Los resultados encontrados en ambos jugos, presentaron buenas características microbiológicas, fisicoquímicas y nutricionales, durante el periodo de almacenamiento estudiado. Por lo tanto, se obtuvieron jugos de umbu con parámetros tecnológicos adecuados y con alto potencial de valorización de esta fruta regional, con características para atender la creciente demanda de alimentos nutritivos y con practicidad para el consumo.

Palabras clave: Biodiversidad; Nutrientes; Compuestos bioactivos; Practicidad.

\section{Introdução}

O Brasil encontra-se na $3^{\text {a }}$ posição no ranking dos maiores produtores mundiais de alimentos. Devido a sua diversidade de frutas tropicais produz, principalmente variedades regionais de importante valor econômico, além das suas principais comodities (CNA, 2017; Rufino et al., 2010). Da produção nacional, cerca de 54\% são destinadas ao mercado de frutas processadas e $46 \%$ são destinadas ao consumo in natura (Sebrae, 2015). O Nordeste se destaca como uma das principais regiões produtoras, com frutos pouco explorados tanto de forma comercial quanto em caráter científico, uma vez que a grande parte destes possuem propriedades relevantes para a saúde da população.

Uma grande variedade de frutas é utilizada para elaboração de sucos naturais, sucos tropicais, integrais e néctares, pois essa é uma forma de oferecer produtos com sabores diversos o ano todo, inclusive de frutas sazonais como o umbu. Assim por serem industrializados, eles têm maior liberdade de consumo no país e no mundo quando comparados às frutas in natura.

Na região Nordeste se encontra o umbu (Spondias tuberosa Arr. Cam.) que é um fruto bastante nutritivo e presente na Caatinga e está integrado na base alimentar e econômica da região (Batista; Silva; Araújo, 2015). Este é um fruto tentador e consumido pelas comunidades locais, o qual é rico em compostos fenólicos e compostos biologicamente ativos (Ribeiro et al, 2017; Lima et al., 2018).

Suco tropical é uma bebida não fermentada obtida pela dissolução da polpa de frutas em água potável ou em suco de fruta tropical clarificado por meio de um processo adequado, o qual deve ter a cor, aroma e sabor característico da fruta para garantir através da técnica de tratamento a aparência e conservação (Decreto $N^{o}$ 6.871, de 4 de junho de 2009). A produção de suco de frutas tropicais, mistos e néctares pode ser uma estratégia interessante para superar as dificuldades de utilização de frutas naturais na elaboração de alimentos valorizando a biodiversidade (Pereira et al., 2016; Santos et al., 2018).

O consumo de suco de frutas e produtos naturais pela população tem aumentado, principalmente devido aos benefícios para a saúde. De acordo com dados divulgados pela Associação Nacional da Agricultura (SNA, 2017), o mercado de sucos no Brasil continua em ascensão, e seu ritmo de crescimento tem superado o do setor de bebida não alcoólicas, evidenciando o enorme potencial de crescimento nos próximos anos. Diante disso a industrialização de frutas tem como objetivo diversificar as possibilidades de comercialização, aumentando sua vida útil e reduzindo perdas pós-colheita (Reis et al., 2017).

Desta forma, a partir da importância do desenvolvimento de novos produtos práticos e nutritivos o objetivo deste trabalho foi desenvolver um suco tropical de umbu e avaliar as características físico-químicas, microbiológicas e compostos bioativos durante 120 dias de estocagem.

\section{Metodologia}

O presente trabalho foi desenvolvido no Instituto Federal de Educação, Ciência e Tecnologia do Sertão Pernambucano, Campus Petrolina-PE, no Laboratório Experimental de Alimentos (LEA). Esse estudo trata-se de uma pesquisa quantitativa na qual faz-se a coleta de dados por intermédio de medições de grandezas e encontra-se valores com suas respectivas unidades. Estes métodos geram dados que podem ser analisados por meio de artifícios matemáticos como porcentagens, estatísticas e probabilidades, além de métodos numéricos, métodos analíticos e geração de equações e/ou fórmulas matemáticas aplicáveis a 
algum processo (Pereira et al., 2018). O experimento foi conduzido em delineamento inteiramente casualizado (DIC) com três repetições para cada tratamento, os quais foram dispostos em esquema fatorial duplo $(2 \times 3)$.

\section{Matéria-Prima}

O fruto Umbu e a polpa congelada foram adquiridos na Cooperativa de Agropecuária Familiar de Canudos, Uauá e Curaçá (Coopercuc), localizada na cidade de Uauá-BA, e foram colhidos nas proximidades da região. Assim, foram trazidos para a cidade de Petrolina-PE. O suco foi obtido de duas formas: utilizando a polpa obtida pronta e congelada e utilizando a polpa obtida do fruto no momento da elaboração do suco.

A obtenção do suco por diferentes matérias-primas teve como objetivo avaliar a ocorrências de alterações significativas nas características físico-químicas e microbiológica dos sucos prontos.

\section{Elaboração do Suco}

Foram escolhidas três formulações para cada matéria-prima (polpa congelada e a polpa do fruto in natura) sendo o suco do umbu obtido pelo despolpado no LEA, denominado Tratamento A, com $35 \%$ de polpa e variações de $12^{\circ} \mathrm{Brix}(\mathrm{A} 1)$, $14{ }^{\circ} \mathrm{Brix}$ (A2) e $16^{\circ}$ Brix (A3). E o suco obtido da polpa congelada do umbu processado pela Coopercuc, denominada Tratamento B, sendo constituída de $35 \%$ de polpa e de $12^{\circ}$ Brix (B1), $14^{\circ}$ Brix (B2) e $16^{\circ}$ Brix (B3).

Figura 1. Elaboração do Suco de Umbu.

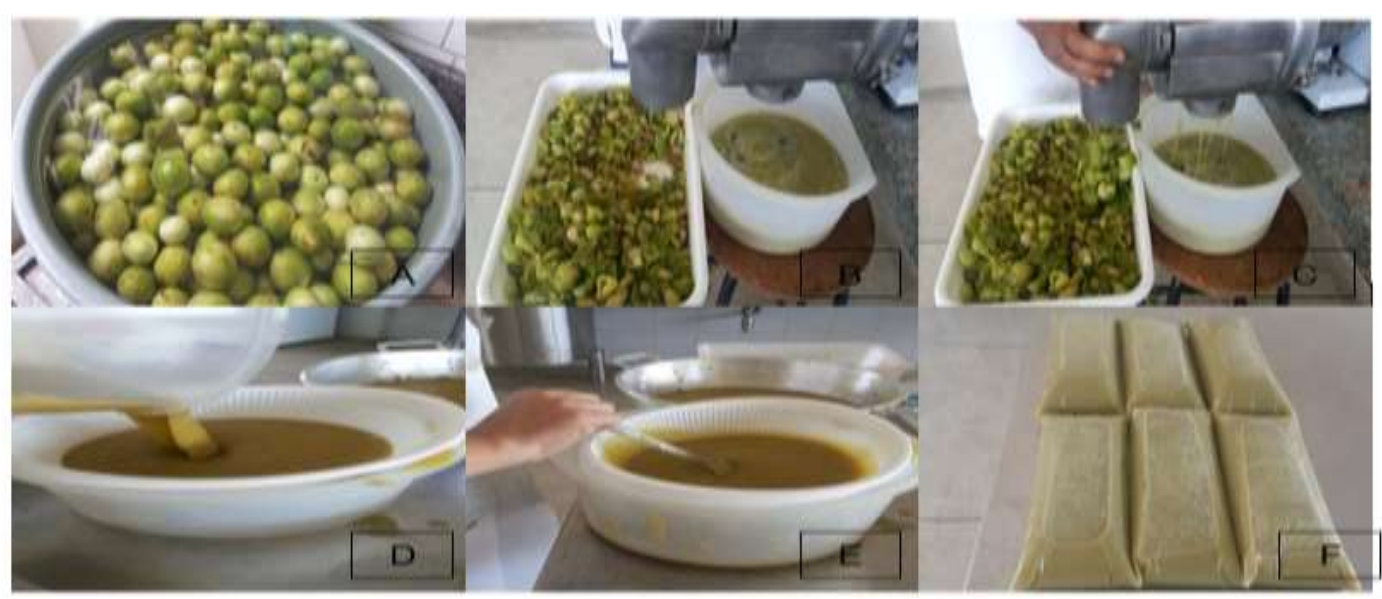

Fonte: Autores (2021).

\section{Definição da formulação}

O balanço de massa foi utilizado para elaboração dos sucos com os diferentes ${ }^{\circ}$ Brix definidos $\left(12,14\right.$ e $16{ }^{\circ}$ Brix $)$. A Equação 1 (um)refere-se ao balanço de massa utilizado para definição das formulações.

Equação 1. Cálculo Balanço de Massa

$\left({ }^{\circ}\right.$ Brix polpa.$\%$ polpa $)+\left({ }^{\circ}\right.$ Brix xarope.$\%$ água $)=\left({ }^{\circ}\right.$ Brix desejado. Vts $)$

Equação 1

onde:

$\mathrm{Vts}=$ volume total do suco. 


\section{Suco do Tratamento A}

Inicialmente foi feito o xarope com a quantidade de água e açúcar determinados na Equação 1. Esse xarope foi levado ao aquecimento apenas para solubilização do açúcar. Após obtenção do xarope e resfriamento até a temperatura ambiente adicionou-se a polpa, e feita a mistura manualmente para não incorporar oxigênio ao suco. Em sequência, fez-se a peneiração para clarificação do suco, com peneiras de tamises 28 e 48 mesh. Após obter o suco clarificado, foi feita uma pasteurização rápida $85^{\circ} \mathrm{C} / 3$ min e envase a quente em garrafas de vidro de $540 \mathrm{~mL}$, previamente esterilizadas.

Figura 2. Fluxograma do Suco Polpa in natura

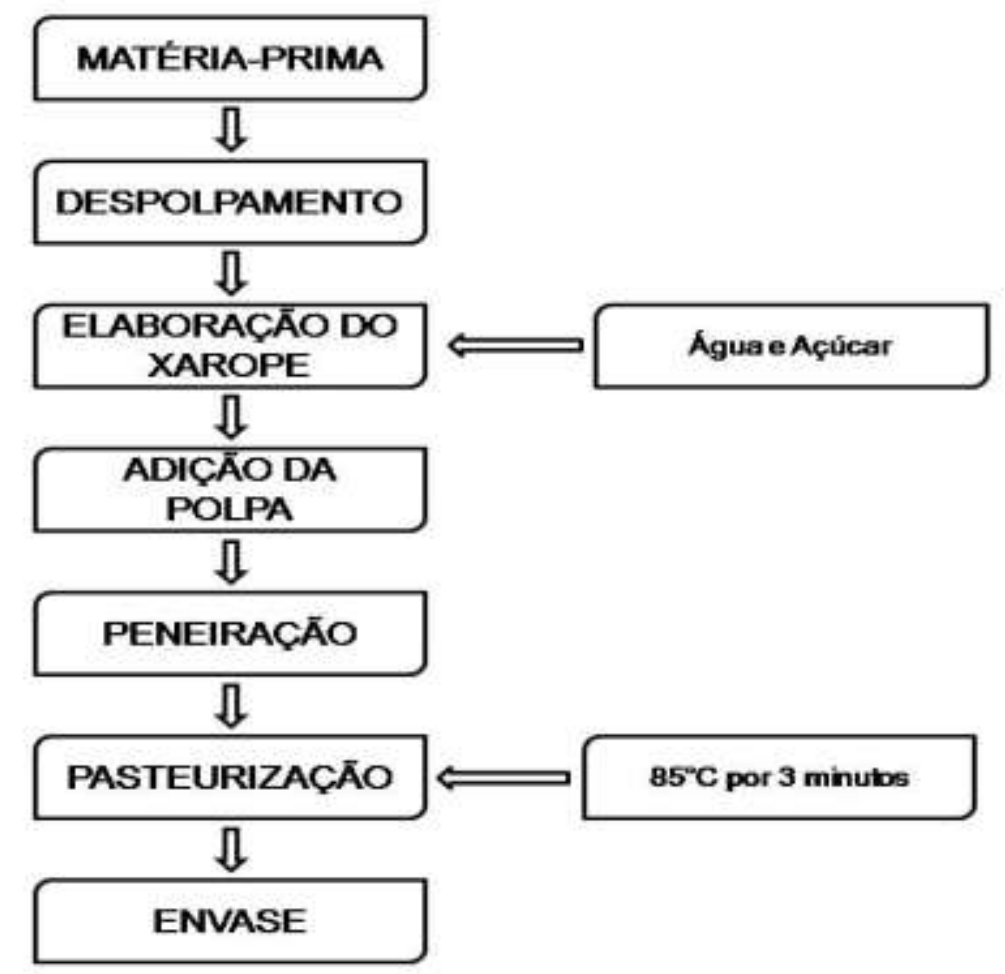

Fonte: Autores (2021).

\section{Suco do Tratamento B}

Para a elaboração do suco foi utilizado o mesmo cálculo de balanço de massa que o suco do Tratamento A. O xarope foi levado ao aquecimento, para total dissolução do açúcar, e reservado até resfriamento a temperatura ambiente. Após este momento, foi adicionado a polpa e feita a mistura manualmente para evitar incorporação de ar. Após obter o suco, foi feita a clarificação em peneiras de tamises 28 e 48 mesh, em sequência, aplicou-se a pasteurização rápida $85^{\circ} \mathrm{C} / 3$ min e envase a quente em garrafas de vidro de $540 \mathrm{~mL}$, previamente esterilizadas. 
Figura 3. Fluxograma Suco Polpa Congelada

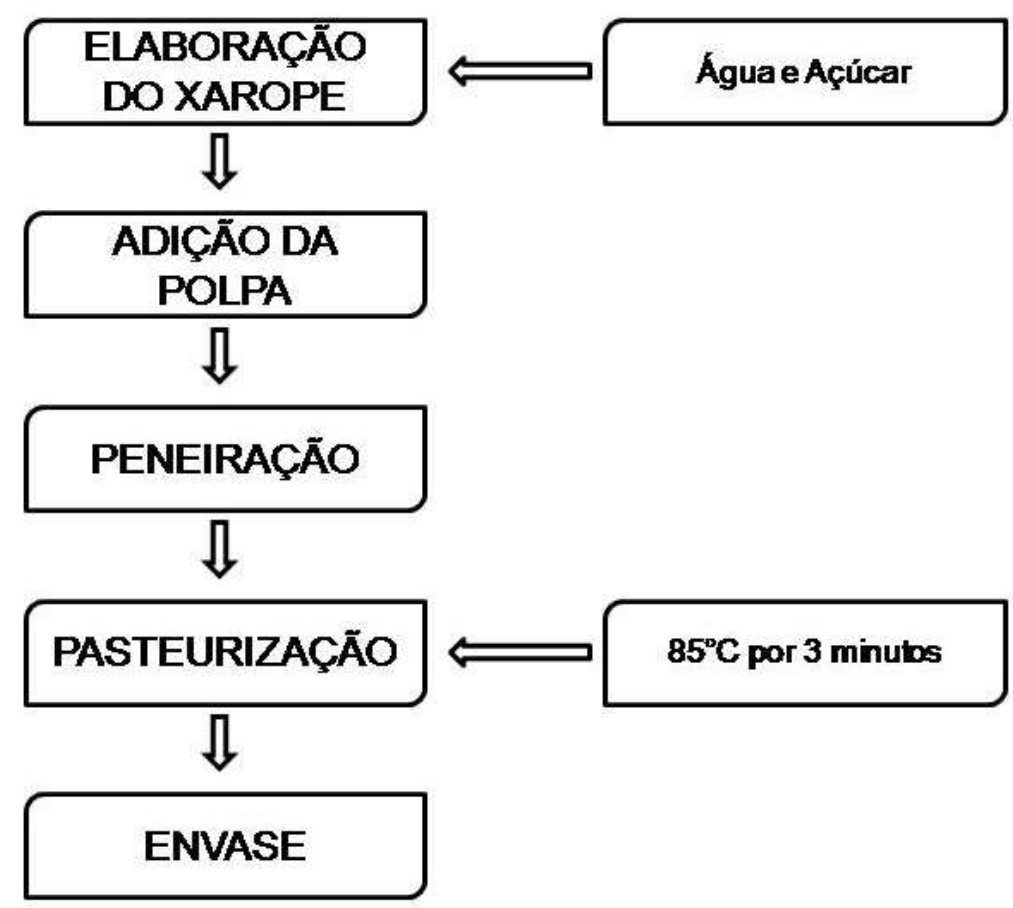

Fonte: Autores (2021).

\section{Análises Microbiológicas}

Após a elaboração dos sucos, foram realizadas as análises microbiológicas: Bolores e Leveduras, Bactérias Totais e E.Coli, seguindo a metodologia de Silva et. al (2010). Seguiram-se também as repetições das análises a cada trinta dias, totalizando cinco meses de acompanhamento.

\section{Caracterização Físico-química}

As análises foram realizadas segundo a metodologia de Instituto Adolfo Lutz (2008), desde o primeiro dia de produção do suco, e as repetições foram feitas a cada trinta dias, totalizando cinco meses de análises. Foram realizadas análise de pH, ${ }^{\circ}$ Brix, acidez total titulável, cor, carboidratos redutores e não-redutores.

\section{Análises de Compostos Bioativos}

Foram realizadas análises de Conteúdo Fenólico Total seguindo a metodologia descrita por Singleton e Rossi (1969) e Determinação da Atividade Antioxidante (AOX), por Captura do Radical Livre DPPH, segundo metodologia Kim et al., 2002 adaptado por Lima et al (2014), durante o período de armazenamento dos dois sucos.

\section{Resultados e Discussão}

Na Tabela 1, estão apresentados os valores médios das análises microbiológicas. 
Tabela 1. Resultados das análises microbiológicas durante o armazenamento.

\begin{tabular}{|c|c|c|c|c|c|c|c|c|}
\hline \multirow{3}{*}{ Análise } & \multicolumn{4}{|c|}{ Tratamento A } & \multicolumn{4}{|c|}{ Tratamento B } \\
\hline & \multicolumn{4}{|c|}{ Tempo de estocagem (dias) } & \multicolumn{4}{|c|}{ Tempo de estocagem (dias) } \\
\hline & 0 & 30 & 60 & 90 & 0 & 30 & 60 & 90 \\
\hline Coliformes totais & 0 & - & - & - & 0 & - & - & - \\
\hline Bactérias totais & 0 & 0 & 0 & 0 & 0 & 0 & 0 & 0 \\
\hline Bolores e Leveduras & 0 & 0 & 0 & 0 & 0 & 0 & 0 & 0 \\
\hline
\end{tabular}

Fonte: Autores (2021).

Os padrões microbiológicos de coliformes, bactérias aeróbias mesófilas, fungos filamentosos e leveduras para bebidas de suco de frutas não são determinados segundo a legislação brasileira RDC n³31/2019 (Brasil, 2019). No entanto, essas análises são muito importantes para verificar a carga microbiana e as condições higiênica-sanitárias do produto que refletem as características de qualidade das matérias-primas, e da higienização dos equipamentos, embalagens e utensílios (Da Matta; Cabral, 2010).

A partir das análises, observou-se que não houve crescimento de bactérias totais, E.Coli, leveduras e bolores. Assim, pode-se concluir que o binômio de tempo e temperatura adotado no tratamento térmico (pasteurização) foi adequado. Como também que o tratamento de esterilização das garrafas foi eficiente para garantir uma longa vida de prateleira aos sucos. Isso demonstra que os sucos desenvolvidos neste trabalho, possuem qualidade higiênica e parâmetros tecnológicos adequados para impedir o surgimento desses microrganismos.

Os resultados da caracterização físico-química dos sucos obtidos pelos Tratamentos A e B são demonstrados nas Tabelas 2 e 3 , logo abaixo: 
Tabela 2. Caracterização parâmetros físico-químicos do Suco de Tratamento A.

\begin{tabular}{|c|c|c|c|c|c|}
\hline \multirow{3}{*}{ Análise } & \multicolumn{5}{|c|}{ Tratamento A } \\
\hline & \multicolumn{5}{|c|}{ Tempo de estocagem (dias) } \\
\hline & 0 & 30 & 60 & 90 & 120 \\
\hline $\mathrm{pH}$ & $2,44 \pm 0,0$ & $2,49 \pm 0,0$ & $2,57 \pm 0,0$ & $2,48 \pm 0,0$ & $2,57 \pm 0,0$ \\
\hline $\begin{array}{l}\text { Acidez Total } \\
\text { Titulável (\%) }\end{array}$ & $0,305 \pm 0,0$ & $0,29 \pm 0,0$ & $0,297 \pm 0,0$ & $0,299 \pm 0,0$ & $0,296 \pm 0,0$ \\
\hline $\begin{array}{c}\text { Sólidos Solúveis } \\
\left({ }^{\circ} \text { Brix }\right)\end{array}$ & $16,03 \pm 0,1$ & $15,7 \pm 0,1$ & $15,733 \pm 0,1$ & $15,7 \pm 0,0$ & $15,6 \pm 0,1$ \\
\hline $\begin{array}{c}\text { Açúcares Redutores } \\
(\%)\end{array}$ & $18,59 \pm 1,4$ & $22,76 \pm 1,0$ & $12,7 \pm 1,0$ & $9,48 \pm 0,9$ & $14,43 \pm 1,5$ \\
\hline $\begin{array}{l}\text { Açúcares Não- } \\
\text { Redutores (\%) }\end{array}$ & - & - & - & - & - \\
\hline
\end{tabular}

Fonte: Autores (2021).

Tabela 3. Caracterização parâmetros físico-químicos do Suco de Tratamento B.

\begin{tabular}{|c|c|c|c|c|c|}
\hline \multirow{3}{*}{ Análise } & \multicolumn{5}{|c|}{ Tratamento B } \\
\hline & \multicolumn{5}{|c|}{ Tempo de estocagem (dias) } \\
\hline & 0 & 30 & 60 & 90 & 120 \\
\hline $\mathrm{pH}$ & $2,42 \pm 0,0$ & $2,45 \pm 0,0$ & $2,53 \pm 0,0$ & $2,44 \pm 0,0$ & $2,54 \pm 0,0$ \\
\hline $\begin{array}{l}\text { Acidez Total } \\
\text { Titulável (\%) }\end{array}$ & $0,343 \pm 0,0$ & $0,34 \pm 0,0$ & $0,344 \pm 0,0$ & $0,348 \pm 0,0$ & $0,352 \pm 0,0$ \\
\hline Sólidos & & & & & \\
\hline $\begin{array}{l}\text { Solúveis } \\
\left({ }^{\circ} \text { Brix }\right)\end{array}$ & $15,27 \pm 0,1$ & $15,3 \pm 0,1$ & $15,333 \pm 0,1$ & $15,17 \pm 0,1$ & $15,3 \pm 0,0$ \\
\hline $\begin{array}{c}\text { Açúcares } \\
\text { Redutores (\%) }\end{array}$ & $16,38 \pm 1,4$ & $18,33 \pm 1,0$ & $12,92 \pm 0,6$ & $15,02 \pm 2,1$ & $15,82 \pm 1,4$ \\
\hline $\begin{array}{l}\text { Açúcares Não- } \\
\text { Redutores (\%) }\end{array}$ & - & - & - & - & - \\
\hline
\end{tabular}


Os sucos de umbu dos tratamentos A e B apresentaram pH durante o período de armazenamento entre 2,44 e 2,57, sendo classificados como alimentos muito ácido. Portanto, fora da zona de perigo para o desenvolvimento de microrganismos patogênicos, como o Clostridium Botulinum, podendo ser considerados seguros do ponto de vista microbiológico. De acordo com Neris et. al (2017) o pH afeta diretamente as características sensoriais e nutricionais do produto, com isso é importante a adição de açúcares em quantidades adequadas para mascarar o gosto ácido de sucos obtidos de frutas com elevada acidez. .

Observou-se que o teor de acidez total titulável não diferiu estatisticamente ao longo dos 120 dias de estocagem, para ambos os tratamentos A e B, aproximadamente 0,30 e 0,34, respectivamente, indicando uma boa estabilidade para a conservação dos sucos de frutas. Alterações na acidez titulável indicam decomposição por hidrólise, oxidação ou fermentação do alimento. Portanto, os parâmetros determinados para o tratamento térmico e o lacre das embalagens deste trabalho podem ser considerados adequados à matéria-prima estudada.

Souza et al. (2017) avaliaram a estabilidade do ácido ascórbico em sucos de frutas, por um período de 21 dias, e observaram uma diminuição da acidez titulável ao longo do período de armazenamento para a maioria dos sucos avaliados.

O teor de sólidos solúveis está relacionado à maturidade e qualidade do fruto, sinalizando alterações físico-química naturalmente ocorridas durante o amadurecimento dos frutos. Os valores encontrados para os sucos obtidos pelos Tratamento A e B, $\sim 15$ e $16{ }^{\circ}$ Brix, respectivamente, não apresentaram diferença significativa, ao nível 5\%. Portanto, o congelamento da polpa da fruta de umbu foi capaz de preservar as qualidades físico-química e sensoriais do fruto. Segundo Chitarra e Chitarra (2005) em muitas frutas o equivalente entre os ácidos orgânicos e os açúcares é utilizado para avaliar o sabor.

Quanto ao teor de açúcares, que tem variação de acordo com cada fruta, avaliou-se que houve uma variação no teor de açúcares redutores no tratamento A e uma melhor estabilidade no tratamento B, e nenhuma quantidade de açúcares não-redutores em ambos os tratamentos. As variações de concentração podem ter ocorrido devido ao grau de maturação dos frutos coletados para o preparo da polpa. Mais uma vez, os resultados demonstram que o congelamento da polpa reduziu as alterações provocadas pelo amadurecimento do fruto. Um elevado grau de maturação resulta no aumento dos teores de açúcares porque as carboidrases presentes nos frutos vão degradando os polissacarídeos, convertendo-os em monossacarídeos e dissacarídeos, entre outros (Chitarra, 2005).

Observa-se na Tabela 4 os valores obtidos para os parâmetros de cor ( $L^{*}, a^{*}$ e b*).

Tabela 4. Colorimetria: Valores da Luminosidade ( $\left.\mathrm{L}^{*}\right)$ e das coordenadas $\left(\mathrm{a}^{*}\right.$ e $\left.\mathrm{b}^{*}\right)$.

\begin{tabular}{|c|c|c|c|c|c|c|}
\hline \multirow{3}{*}{ Dias } & \multicolumn{3}{|c|}{ Tratamento A } & \multicolumn{3}{|c|}{ Tratamento B } \\
\hline & \multicolumn{3}{|c|}{ Parâmetros } & \multicolumn{3}{|c|}{ Parâmetros } \\
\hline & $\mathrm{L}^{*}$ & $a^{*}$ & $\mathrm{~b}^{*}$ & $\mathrm{~L}^{*}$ & $a^{*}$ & $b^{*}$ \\
\hline 0 & $47,44 \pm 0,834$ & $2,77 \pm 0,080$ & $28,13 \pm 0,390$ & $43,59 \pm 0,4$ & $3,63 \pm 0,021$ & $34,15 \pm 0,232$ \\
\hline 30 & $47,60 \pm 0,295$ & $1,91 \pm 0,006$ & $28,73 \pm 0,076$ & $43,51 \pm 0,420$ & $3,44 \pm 0,038$ & $34,27 \pm 0,323$ \\
\hline 60 & $48,09 \pm 0,520$ & $1,57 \pm 0,092$ & $31,03 \pm 0,444$ & $40,77 \pm 0,717$ & $3,13 \pm 0,052$ & $32,26 \pm 0,598$ \\
\hline 90 & $49,03 \pm 0,812$ & $2,57 \pm 0,160$ & $30,66 \pm 0,626$ & $38,77 \pm 0,079$ & $3,35 \pm 0,040$ & $29,47 \pm 0,072$ \\
\hline 120 & $47,21 \pm 0,233$ & $1,95 \pm 0,040$ & $30,58 \pm 0,167$ & $42,81 \pm 0,903$ & $4,44 \pm 0,121$ & $34,74 \pm 0,578$ \\
\hline
\end{tabular}

Fonte: Autores (2021). 
De acordo com os resultados demonstrados na Tabela 4, houve pequenas alterações na coloração de ambos os sucos. Porém, as alterações demonstradas nos parâmetros $\mathrm{a}^{*} \mathrm{e} \mathrm{b}^{*}$ dos dois tratamentos, durante os noventa dias de armazenamento, não foram demasiadamente significativa. Patras (2019), afirma que geralmente as mudanças de cor podem estar relacionadas ao $\mathrm{pH}$ da matéria prima, mas também devido à polimerização e escurecimento dos pigmentos, por aplicação de processos térmicos. Araújo et al. (2020), após analisarem a polpa de umbu, mostraram que mesmo não apresentando diferença significativa entre os tratamentos houve uma predominância da cor amarela.

Os resultados para os compostos bioativos dos sucos obtidos da fruta de umbu in natura e da polpa congelada podem ser observados na Tabela 5 .

Tabela 5. Valores de Compostos bioativos durante o armazenamento dos sucos.

\begin{tabular}{|c|c|c|c|c|}
\hline \multirow{2}{*}{ Dias } & \multicolumn{2}{|c|}{ Tratamento A } & \multicolumn{2}{|c|}{ Tratamento B } \\
\hline & $\begin{array}{c}\text { Fenólicos Totais (mg } \\
\text { ác.gálico/L) }\end{array}$ & $\mathrm{AOX}$ & $\begin{array}{c}\text { Fenólicos Totais (mg } \\
\text { ác.gálico/L) }\end{array}$ & $\mathrm{AOX}$ \\
\hline 0 & - & - & - & - \\
\hline 30 & $303,5 \pm 20$ & $35,7 \pm 2,5$ & $433,9 \pm 22,1$ & $51,2 \pm 0,6$ \\
\hline 60 & $271,4 \pm 2,1$ & $29,6 \pm 2,6$ & $407,9 \pm 24,4$ & $38,2 \pm 6,9$ \\
\hline 90 & $271,4 \pm 4,2$ & $26,4 \pm 1,9$ & $423,4 \pm 25,1$ & $39,8 \pm 1,5$ \\
\hline 120 & $300,5 \pm 22,9$ & $31,7 \pm 2,2$ & $441,1 \pm 25,7$ & $51,6 \pm 0,9$ \\
\hline
\end{tabular}

Fonte: Autores (2021).

O suco obtido da polpa congelada apresentou uma quantidade de compostos fenólicos totais e atividade antioxidante maiores do que o do suco in natura. Essa diferença pode estar relacionada às características dos diferentes lotes das matériasprimas, como também pode indicar que o congelamento aplicado à polpa, logo após o despolpamento, foi capaz de preservar as propriedades físico-químicas deste fruto. No entanto, para ambos os sucos, os compostos bioativos foram mantidos durante o armazenamento.

A partir da análise dos compostos bioativos observou-se que há a presença desses compostos nos sucos desenvolvidos, o que resultam em um produto com um valor agregado superior aos demais produtos similares do mercado. Costa et al. (2015) ao avaliarem o resíduo de umbu encontraram o valor de $776 \mathrm{mg}$ ác.gálico/L, sendo este superior ao do presente estudo em ambos os tratamentos, essa diferença é atribuída ao fato do aquecimento no processo de pasteurização que ocasionou na degradação dos compostos.

Devido a quantidade significativa de compostos fenólicos, observou-se que os sucos em ambos os tratamentos possuem capacidade antioxidante. Santos e Silva (2018) ao avaliarem cincos genótipos de umbu encontraram valores próximos ao do presente estudo, variando entre 38,8 e 44,5 $\mu \mathrm{Mol}$ TEAC L ${ }^{-1}$, observando assim que há uma correlação entre a quantidade de compostos fenólicos com a capacidade antioxidante. Assim a utilização do fruto umbu na elaboração dos sucos, mostrou-se eficiente mesmo passando pelo processo de pasteurização apresentou quantidades significativas desses compostos.

Assim, o fruto de umbu in natura ou congelado pode ser utilizado para obtenção de um suco tropical seguro, nutritivo e economicamente viável, por meio de processo tecnológico adequado, e com potencial praticidade para o consumo. 


\section{Considerações Finais}

O umbu é uma fruta rica em compostos bioativos e nutrientes essenciais para saúde humana, porém pouco explorada, este estudo demonstrou que ela pode ser utilizada para elaboração de produtos alimentares, como por exemplo sucos tropicais, devido a sua rica composição química riqueza, por adequação aos processos industriais e por levar praticidade e facilidade de consumo desta fruta exótica para a população.

Com isso, por meio dos resultados obtidos referentes à estabilidade físico-química, microbiológica e de compostos bioativos do suco de umbu em ambos os tratamentos, pode-se concluir que todas as condições de higienização, processamento e armazenamento se apresentaram satisfatórias e adequadas para obtenção de um produto de maior valor agregado para esta fruta regional.

Aconselha-se a utilização de outros tratamentos e de maiores tempos de armazenagem, bem como o uso de outras técnicas de processamento, uma vez que o umbu é uma variedade pouco explorada na região do Submédio do Vale do São Francisco e apresenta boas propriedades para a formulação de novos subprodutos.

\section{Agradecimentos}

Ao Instituto Federal do Sertão Pernambucano (IF-Sertão), pela concessão da bolsa de iniciação científica e a todos parceiros envolvidos neste estudo.

\section{Referências}

Araújo, E. J. S., Santos dos, J. A. B., \& Narain, N. (2020). Avaliação da influência de diferentes condições de liofilização nas características físico-químicas e sensoriais do umbu em pó. Brazilian Journal of Development.

Batista, F. R. C., Silva, M. M. A., \& Araújo, V. S. (2015). Uso sustentável do umbuzeiro: estratégia de convivência com o semiárido. INSA - Instituto Nacional do Semiárido. $15 \mathrm{p}$.

Brasil - Agência Nacional de Vigilância Sanitária (2019). Regulamento Técnico sobre Padrões microbiológicos para alimentos, RDC N³31 de 23 de dezembro de 2019.

Brasil - Instituto Adolfo Lutz. (2008). Métodos físico-químicos para análise de alimentos. (4a ed.), 1020p.

Brasil. Decreto No 6.871, de 4 de Junho de 2009. MAPA. (2009). Padronização, classificação, registro, inspeção, produção e fiscalização de bebidas. Brasília, DF.

Campos, A. N. da R., Pena, S. de M., Trevizano, L. M., Caetano, F. B., Moreira, L. A., \& Mattos, L. N. (2016). Ciência e Tecnologia no Campus Rio Pomba do IF Sudeste MG: contribuições para a Zona da Mata Mineira. 1, 6-25.

Chitarra, M. I. F. \& Chitarra, A. B. (2005). Pós-colheita de frutos e hortaliças: fisiologia e manuseio. (2a ed.) UFLA.

CNA - Confederação da Agricultura e Pecuária do Brasil. Fruticultura: Balanço 2016. Perspectivas 2017. http://www.cnabrasil.org.br/sites/defa ult/files/sites/default/files/uploads/10_fruticultura.pdf.

Costa, F. I. B., Porfírio, M. C.P., Oliveira, J. B., Santana, G. A., Lage, R. S., \& Silva, M. V. (2015). Avaliação Fitoquímica e Screening da Capacidade Antioxidante de Resíduos de Umbu. Revista Brasileira de Produtos Agroindustriais.17(4), 341-348.

Da Matta, V. M., \& Cabral, L. M. C. Suco de acerola. In: Venturini Filho, W. G. (2010). Bebidas não alcoólicas: Ciência e tecnologia. Blucher, $12.215-225$.

Dos Santos, C. M., \& Silva, F. V. G. (2018). Qualidade Pós-Colheita de Frutos de Genótipos de Umbuzeiro das Mesorregiões da Borborema e do Agreste da Paraíba. Trabalho de conclusão de curso.

Kim, Y K., Guo, Q., \& Packer, L. (2002). Free radical scavenging activity of red ginseng aqueous extracts. Toxicology, 172, $149-156$.

Lima, M. A. C., Silva, S. M. \& Oliveira, V. R. (2018). Umbu— Spondias tuberosa. Exotic Fruits, 1(1), 427-433.

Lima, M. S., Silani, I. S. V., et al. (2014). Phenolic compounds, organic acids and antioxidant activity of grapes juices produced from new Brazilian varieties planted in the Northeast Region of Brazil. Food Chemistry, 161, 94-103.

Martins, H. D., Perfeito, D. G. A., Silva, A. R., \& Peixoto, N. (2017). Caracterização e estudo da estabilidade física de suco misto adoçado de mangaba e cagaita. Revista de Agricultura Neotropical, 4(2), 81-87. 
Neris, T. S., Loss, R. A., \& Guedes, S. F. (2017). Caracterização físico-química da seriguela (Spondias purpurea L.) colheitadas no município de Barra do Bugres/MT em diferentes estágios de maturação. Natural Resources, 7(1), 9-18. http://doi.org/10.6008/SPC2237-9290.2017.001.0002

Nielsen. (2016). Quase 7 em cada 10 brasileiros estão dispostos a pagarem mais por alimentos e bebidas que não contêm ingredientes indesejáveis. Tendências Globais. http://www.nielsen.com/br/pt/press-room/2016/Quase-7-em-cada-10-brasileiros-estao-dispostos-a-pagar-mais-por-alimentos-e-bebidas-semingredientes-indesejaveis.html

Patras, A. (2019). Stability and colour evaluation of red cabbage waste hydroethanolic extract in presence of different food additives or ingredients. Food chemistry. 275, 539-548.

Pereira, A. S., Shitsuka, D. M., Parreira, F. J., \& Shitsuka, R. (2018). Metodologia da Pesquisa Cientifica. UFSM.

Pereira, D. C. de S., Campos, A. N. da R., Martins, E. M. F., \& Martins, M. L. (2016). Utilização dos frutos da palmeira juçara (Euterpe edulis Martius) como estratégia para conservação da espécie e alternativa de renda para o município de Rio Pomba. Minas Gerais.

Reis, D. S., Neto, A. F., Ferraz, A. V., \& Freitas, S. T. (2017). Production and storage stability of acerola flour dehydrated at different temperatures. Braz. J. Food Technol., 20, e2015083.

Ribeiro, L. O., Pontes, S. M., Ribeiro, A. P. O., Pacheco, S., Freitas, S. P., \& Matta, V. M. (2017). Avaliação do armazenamento a frio sobre os compostos bioativos e as características físico-químicas e microbiológicas do suco de umbu pasteurizado. Brazilian Journal of Food Technology, 20(3), 1-8.

Romano, K., R., Rosenthal, A., \& Deliza, R. (2015). How do Brazilian consumers perceive a non-traditional and innovative fruit juice? An approach looking at the packaging. Food Research International, 74, 123-130.

Rufino, M. do S. M., Alves, R. E., de Brito, E. S., Pérez-Jiménez, J., Saura-Calixto, F., \& Mancini-Filho, J. (2010). Bioactive compounds and antioxidant capacities of 18 non- traditional tropical fruits from Brazil. Food Chemistry, 121, 996-1002.

Santos, G. Z., Campos, A. N. da R., Martins, E. M. F., \& Silva, V. R. O. (2018). Aceitação sensorial de bebidas mistas de acerola com juçara ao longo do tempo de estocagem. Higiene Alimentar, 32, 82-86.

Sebrae. Boletim de inteligência agronegócio fruticultura. (2015). http://www.sebraemercados.com.br/wp-content/uploads/2015/11/Panorama-do-mercado--defruticultura-no-Brasil.pdf

Silva, N., Junqueira, V. C. A., Silveira, N. F. A., Taniwaki, M. H., Santos, R. F. S., \& Gomes, R. A. R. (2010). Manual de Métodos de Análise Microbiológica de Alimentos e ÁguaLivraria Varela, (4a ed.),

Singleton, V. L., \& Rossi, J. A. (1965). Colorimetry of total phenolics with phosphomolybdic phosphotungstic acid reagents. American Journal of Enology and Viticulture, 16, 144-158.

Souza et al. (2017). Avaliação físico-química e estabilidade do ácido ascórbico em sucos de frutas comercializados no município de Frutal, Minas Gerais. Revista Verde de Agroecologia e Desenvolvimento Sustentável. 12(14). 